\title{
Detection of Primary User in Wide-band Cognitive Radio Networks over Fading Channel using Compressed Sensing
}

\author{
Sabah M. Ahmed \\ Electrical and Electronics \\ Engineering Department, Faculty of \\ Engineering, Assiut University, Egypt. \\ Mechatronics and Robotics \\ Engineering Department, School of \\ Innovative Design Engineering, Egypt- \\ Japan University for Science and \\ Technology, Alexandria, Egypt.
}

\author{
M. Abo-Zahhad \\ Electrical and Electronics \\ Engineering Department, Faculty of \\ Engineering, Assiut University, Egypt. \\ Electronics and Communication \\ Engineering Department, School of \\ Electronics, Communications and \\ Computer Engineering, Egypt-Japan \\ University for Science and Technology, \\ Alexandria, Egypt. \\ Khaled Ali BaAli \\ Electrical and Electronics \\ Engineering Department, Faculty of \\ Engineering, Assiut University, Egypt. \\ Electronics and Communication \\ Department, Faculty of Engineering \\ and Petroleum, Hadhramaut University, \\ Yemen.
}

\author{
Mohammed Farrag \\ Electrical and Electronics \\ Engineering Department, Faculty of \\ Engineering, Assiut University, Egypt. \\ Electrical Engineering Department, \\ King Khalid University (KKU), Abha, \\ KSA.
}

\begin{abstract}
In wideband cognitive radio networks, Nyquist sampling rate is very challenging problem. It required expensive high speed analog to digital converter and large storage spaces. Lately, compressive sensing has been emerged as significant solution to crack the conventional sampling rate requirements. It proved the ability to sample below Shannan-Nyquist criteria and reconstructing back the signal after considerable dimensional reduction. Mostly in cognitive radio networks, energy detection is widely used due to its simple implementation and blind detection property. However, regardless that energy detection is subject to noise uncertainty as well as shadowing and fading which deteriorate its detection performance. Several articles have been published to improve energy detection performance using large number of measurements. In this paper, since, the detection performance using small number of measurements or compressed measurements achieved significant performance using energy detection under additive white Gaussian noise channel. This motivated us to investigate the performance of compressed measurements-based detection over fading channels which has not been studied yet. The proposed algorithm has been implemented using MATLAB. We also studied the tradeoff between the compression ratios and using fraction of transmitted signal and its
\end{abstract}

impact on detection performance and threshold choice. In comparison with the ordinary compressed energy detection over the Rayleigh fading channel the results reveal that the proposed enhanced compressed measurements-based energy detection is better in performance of detection.

\section{Introduction}

Because of a deficiency of spectrum, Cognitive Radio (CR) has been suggested as a tempting means to alleviate the problem. Empirical investigations have been conducted by Federal Communications Commission (FCC) and other agencies. They have come to the conclusion that more than $70 \%$ of the spectrum were poorly used because of a scarcity of spectrum [1-3]. To overcome this problem, they suggested opening unused licensed frequency channels by for primary users (PUs) to be shared with unlicensed ones (i.e., secondary users (SUs)). These unused licensed frequency channels commonly known as spectrum holes - these are based on the opportunistic basis either by using overlay or underlay methods [4].

CR has been adopted to make the SUs access the unused licensed frequency bands easily. The access to these hidden spectral opportunities could be achieved by exploiting spectrum sensing without interfering with the PUs communications as well as the other neighboring. As 
some services moved from analog to digital broadcasting TV, e.g., VHF/UHF bands, they are proposed by IEEE 802.22 wireless regional area network to be used by CR networks with sensing time less than two seconds, the probability of detection equal $90 \%$ and the probability of false alarm equal $10 \%$ or less. CR transceiver sensitivity is $-21 \mathrm{~dB}$ for digital and $-95 \mathrm{~dB}$ for analog signals [5].

Due to conventional sampling rate requirements, sensing becomes a very challenging task, for example, we require 12 Giga sample per second (Gsps) to sample $6 \mathrm{GHz}$ frequency band), something that may prove too costly. To settle the matter, Compressive sensing (CS) has been energetically proposed as one of the best solutions. It is a powerful mathematical tool that proved the ability of sampling below the conventional Nyquist sampling condition and recovering the transmitted signal once again after great dimensional reduction at the reception side by using reconstruction algorithms $[6,7]$.

Spectrum sensing performance could be measured by means of two metrics: the false alarm probability and the detection probability. The former is when a frequency of a certain band is declared to be not free while it is. The latter is when the frequency of a certain band is successfully declared to be occupied when it is. Various spectrum detection techniques have been adopted in literature such as energy detector, cyclo stationary detector and so on. Among spectrum sensing techniques, energy detector is the most commonly employed due to its simple implementation and blind property.

Despite its advantages, energy detector is prone to noise uncertainty, shadowing and fading, and, as a result decrease in its detection performance. it is an accepted fact that the analytic expressions of detection and false alarm probability have firstly been addressed and derived by Urkowitz [8]. Then intensive efforts have been made to improve the performance of detection of energy detector [9-15]. Lately, the wideband spectrum sensing problem has been reviewed over fading channels exploiting energy detection [16-23].

Consequently, several mathematical expressions were derived [17, 24-29]. Nevertheless, these expressions have been influenced by errors of truncation. de Carvalho et al [19] studied analytically the effect of numerous fading models on the performance of detection of CR sensing task by means of energy detector. They suggested exploiting energy detector on channel subjected to lognormal fading as it achieved well performance than other channels. Atawi et al reported that the relationship between probability of detection and time-bandwidth product is inversely proportion using generalized-K (EGK) distribution [20].

The proposed analytic expression can be performed well exploiting integer and non-integer values of fading parameters for reception modes, non-diversity, and diversity. Considering the effect of multiple PUs on the performance of both probabilities of detection and false alarm, a innovative closed form of detection probability is provided [21]. To deal with the fading channel effect, [22] proposed cooperative sensing approach. In this approach, wideband spectrum is segmented into different separate narrowband clusters to be sensed by multiple SUs over channels of small-scale fading. In each cluster, cluster head responsible for sending the decision about frequency band status to the fusion center has been nominated. An alternative to Marcum Q-function approximation is used in in [23]. They provided different analytical approach to express the detection probability over different fading channels.

Further improving on has been achieved to detection performance of energy detector by employing CS over Additive White Gaussian Noise (AWGN) channel [30, 31]. To the extent that the researchers' attention, the expression of the detection probability based on compressed measurements over fading channel has not been experimentally derived yet. Based on that, the current study attempts to attain a derivation for the aforementioned expressions using energy detector. Our contribution is to show that despite the reduction of using a small number of measurements or collected samples $M(M \square N)$, the energy detector based compressed measurements can obviously able to detect the presence of PU transmitted signal on the observed frequency band in fading channel as well in AWGN. In compare to the traditional case without the use of compressed sensing, the detector requires large number of measurements or collected samples $N(M \rightarrow N)$ to detect the existing of PU signal on that channel under observation.

Which by result, using less measurements $M$ for detection is much better than using more measurements $N$ in the same case in which this reduction contribute implicitly faster detection with less time. In addition to that in traditional energy detector using less measurements may cause falsely declaring the observed channel unoccupied while it is not and yields harmful interference to the network. Therefore, in this work, we extended the use of less measurements in compressed measurement based energy detection (CMBED) after enhancing its performance as showed by simulation results to include the case of fading channel, e.g., Rayleigh fading channel. The rest of the paper is organized as system model and proposed work are discussed in section 2 and 3 respectively. In section 2 , we described the signal used in the proposed framework. While in section 3, we derived the probability of detection and false alarm over both AWGN and Rayleigh fading channels Simulation results are highlighted in section 4 . While our work is concluded in section 5 .

\section{System Model}

The conventional objective of spectrum detection of deterministic signal could be formulated as test of binary hypothesis to prove the existence of PU transmitted signal on the observed frequency band. 


$$
x(t)= \begin{cases}n(t) & : \mathrm{H}_{0}, \mathrm{PU} \text { is absent } \\ h(t) * s(t)+n(t): \mathrm{H}_{1}, \mathrm{PU} \text { is present }\end{cases}
$$

where $x(t)$ is the received signal by the SU, $s(t)$ is the PU signal with bandwidth $W$ in which it is an independent and identical Gaussian random variable. $n(t)$ is AWGN with mean is zero and variance is unity, $\mathrm{N}(0,1) \cdot \mathrm{H}_{1}$ and $\mathrm{H}_{0}$ denote the presence and absence of the PU transmitted signal respectively. $h$ is the channel coefficient and presumed as time invariant during the sensing period. The contained energy in the compressed measurements could be given as:

$$
\xi_{N}=\sum_{k=0}^{N-1}\left(x_{k} / \sigma\right)^{2}
$$

where $x_{k}$ is the samples collected from the signal under observation and $\sigma^{2}$ is the variance of noise. The decision $\xi_{N}$ assumed to follow central chi-square distribution under $\mathrm{H}_{0}$ and noncentral chi-square distribution with $N$ degrees of freedom under $\mathrm{H}_{1}$ could be expressed as:

$$
\xi_{N} \square \begin{cases}\chi_{N}^{2} & : \mathrm{H}_{0} \\ \chi_{N}^{\prime 2}(\mu) & : \mathrm{H}_{1}\end{cases}
$$

The chi-square distribution pdf could be given as:

$$
f_{x}(x)= \begin{cases}\frac{1}{\sigma^{2} 2^{N / 2} \Gamma(N / 2)} x^{(N / 2-1)} \exp \left(-x / \sigma^{2}\right) & : \mathrm{H}_{0} \\ \frac{1}{2}\left(\frac{x}{\mu}\right)^{(N-2 / 4)} \exp (-\mu+x / 2) I_{N / 2-1}(\sqrt{\mu x}) & : \mathrm{H}_{1}\end{cases}
$$

where $\Gamma(\cdot)$ is gamma function, $I_{v-1}(\cdot)$ is the modified Bessel function of the first kind and $\mu$ is noncentrality parameter and $x_{k}$ is the kth Gaussian random variable. The statistical test $\xi_{N}$ is compared to predetermine threshold $\lambda$. Hence, false alarm probability and detection probability could be obtained as follows:

$$
P_{F}=\operatorname{Pr}\left(x>\lambda \mid \mathrm{H}_{0}\right)=\int_{\lambda}^{\infty} f_{x, \mathrm{H}_{0}}(x) \mathrm{d} x=\int_{\lambda}^{\infty} \frac{1}{\sigma^{2} 2^{N / 2} \Gamma(N / 2)} x^{(N / 2-1)} \exp \left(-x / \sigma^{2}\right) \mathrm{d} x
$$

$$
P_{D}=\operatorname{Pr}\left(x>\lambda \mid \mathrm{H}_{1}\right)=\int_{\lambda}^{\infty} f_{x, \mathrm{H}_{1}}(x) \mathrm{d} x=\int_{\lambda}^{\infty} \frac{1}{2}\left(\frac{x}{\mu}\right)^{(N-2 / 4)} \exp (-\mu+x / 2) I_{N / 2-1}(\sqrt{\mu x}) \mathrm{d} x
$$

and after some manipulations on (5) and (6), we come to the following findings:

$$
\begin{aligned}
& P_{F}=\Gamma\left(N / 2, \lambda / 2 \sigma^{2}\right) / \Gamma(N / 2) \\
& P_{D}=Q_{N / 2}\left(\sqrt{\mu / \sigma^{2}}, \sqrt{\lambda / \sigma^{2}}\right)
\end{aligned}
$$

In the following section, we will discuss our proposed work on how to derive the probability of detection based on compressed measurements under AWGN and over Rayleigh fading, i.e., a special case of Nakagami-m fading channel with $m=1$.

\section{Proposed work}

In this section, first, we analyzed the probability of detection and false alarm over AWGN channel. Second, we derived the same probabilities over Nakagami-m with $\mathrm{m}=1$ fading channel using compressed measurements. Compressivelyin vector notation, the problem ofdetection could be expressed as follows:

$$
\mathbf{y}=\mathbf{A x}
$$

Where $\mathbf{x} \in \square^{1 \times N}$ is the received signal, $\mathbf{y} \in \square^{M \times 1}$ is the compressed measurements vector and $\mathbf{A} \in \square^{M \times N}$ is the measurement (sensing) matrix. The PU signal xis sensed by using CS unit to get the compressed measurements ywith low dimensionality as depicted in Fig. 1. In [31, 32], by using the compressed measurements $\mathbf{y}$, the detection decision is defined as $\mathrm{H}_{1}$ if the $\mathrm{PU}$ signal is detected else $\mathrm{H}_{0}$ is announced. The system showed a dramatic reduction in computational cost due to the reduce in signal dimensionality.

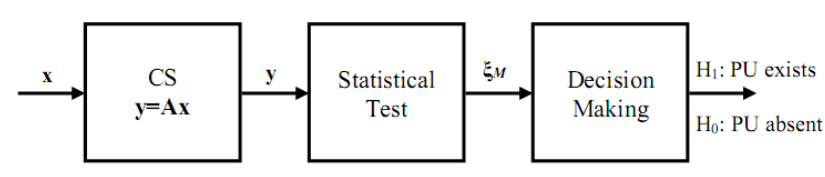

Figure 1. Compressed measurements-based energy detection system.

DCT is used as a measurement (sensing) matrix. It is, explicitly, a sequence of finite data points in terms of the sum of cosine functions oscillating at different frequencies. The entry of measurement matrix is placed at the $k^{\text {th }}$ row and $i^{\text {th }}$ column and the DCT first $M$ rows are chosen according to the coefficients with high-energy. The decision has been achieved after the energy of signal is computed from the compressed measurements as stated by Parseval's theorem as follows [8, 31]:

$$
\xi_{M}=\sum_{k=0}^{M-1}\left(y_{k} / \sqrt{N_{0} W}\right)^{2}
$$

where $N_{0}$ is the power of noise. Based on the amount of $\xi_{M}$ compared to a pre-determined threshold $\lambda$, the decision would be chosen $\mathrm{H}_{0}$ or $\mathrm{H}_{1}$ as follows:

$$
\xi_{M} \underset{\mathrm{H}_{0}}{\gtrless} \lambda
$$

\subsection{Detection and False Alarm Probability over AWGN channel}

For the simple non-fading channel, i.e., AWGN, the observable signal in vector notation in (1) is expressed as:

$$
\mathbf{x}= \begin{cases}\mathbf{n} & : \mathrm{H}_{0} \\ \mathbf{s}+\mathbf{n} & : \mathrm{H}_{1}\end{cases}
$$


Under hypothesis $\mathrm{H}_{0}$, the received signal is noise, $n \stackrel{\text { iid }}{\square} \mathrm{N}\left(0, \sigma^{2}\right), n_{k}=\left[n_{0}, n_{l}, \ldots, n_{M-1}\right]$.The signal energy is computed as:

$$
\xi_{M}=\sum_{k=0}^{M-1}\left(n_{k} / \sigma\right)^{2}
$$

Similarly, under the hypothesis $\mathrm{H}_{1}, x_{k}=\left[s_{0}+n_{0}, s_{I}+n_{l}\right.$, $\left.\ldots, s_{M-I}+n_{M-1}\right]$ and the signal energy is expressed as:

$$
\xi_{M}=\sum_{k=0}^{M-1}\left(s_{k}+n_{k} / \sigma\right)^{2}
$$

where $\sigma^{2}=N_{0} W$, the decision of (14) follows noncentral chi-square distribution as it is illustrated in (4) under with $M$ degrees of freedom and $\mu$ is noncentrality parameter which is related to signal-to-noise ratio, expressed as:

$$
\mu=\sum_{k=0}^{M-1}\left(s_{k} / \sigma\right)^{2}=\left(1 / \sigma^{2}\right)_{k=0}^{M-1}\left(s_{k}\right)^{2}
$$

with DCT as sensing measurements, we use $M$ coefficients out of $\mathrm{Nin}$ the conventional scenario of signal energy calculation $E_{s}$ as (2). In other words, we use a fraction of the energy contained in the signal as in:

$$
\eta_{E}=\left(1 / E_{s}\right) \sum_{k=0}^{M-1}\left(s_{k}\right)^{2}
$$

Substituting (16) for (15) yields:

$$
\mu=\sum_{k=0}^{M-1}\left(s_{k} / \sigma\right)^{2}=\eta_{E} E / \sigma^{2}=\eta_{E} \gamma
$$

where $\gamma$ is signal-to-noise ratio, $\eta_{E}=E_{s} / \sigma^{2}$ again substituting (17) for (8), the result is:

$$
P_{D}=Q_{M / 2}\left(\sqrt{\eta_{E} \gamma}, \sqrt{\lambda}\right)
$$

in which the same equation has already been derived in [31] assuming $\sigma^{2}=1$.

\subsection{Detection and false alarm Probability over fading channel}

The average signal-to-noise ratio over fading effect is given as $\bar{\gamma}=h^{2} \mu / \sigma^{2}[16,34]$ and the energy content of signal under $\mathrm{H}_{1}$ is presented as follows:

$$
\xi_{M}=\sum_{k=0}^{M-1}\left(h_{k} s_{k}+n_{k} / \sigma\right)^{2}
$$

If we assume the channel coefficient to be time invariant during the observation interval, then $h_{k} \rightarrow h$. The noncentrality parameter is given as:

$$
\mu=\sum_{k=0}^{M-1}\left(h_{k} s_{k} / \sigma\right)^{2}=\left(h^{2} / \sigma^{2}\right)^{M-1}\left(s_{k}\right)^{2}
$$

using the similar steps as in AWGN channel, the fraction of that energy contained in the signal is:

$$
\eta_{E}=\left(1 / E_{s}\right) \sum_{k=0}^{M-1}\left(s_{k}\right)^{2}
$$

By substituting (21) for (20), we get:

$$
\mu=\sum_{k=0}^{M-1}\left(h s_{k} / \sigma\right)^{2}=h^{2} \eta_{E} E / \sigma^{2}=h^{2} \eta_{E} \gamma
$$

Finally, substituting (22) for (8) produces:

$$
P_{D}=Q_{M / 2}\left(\sqrt{h^{2} \eta_{E} \gamma}, \sqrt{\lambda}\right)
$$

The probability density function of Nakagami- $m$ could be expressed as central chi-square distribution[35] and
Rayleigh channel probability distribution function is considered as a special case of Nakagami-m, $\mathrm{m}=1$, and is presented as:

$$
f_{\gamma, N a k}=\frac{1}{\bar{\gamma}} \exp \left(-\frac{\gamma}{\bar{\gamma}}\right)
$$

The conventional method for obtaining the average $P_{D}$ over the distribution of specific fading is by averaging $P_{D}$ under AWGN over $f_{\gamma}(\gamma)$ of the output SNR and this method could be expressed as:

$$
\bar{P}_{D B C M}=\int_{0}^{\infty} P_{D}(\gamma) f_{\gamma}(\gamma) d \gamma
$$

where $f_{\gamma}(\gamma)$ is the distribution of fading channel as in:

$$
\bar{P}_{D B C M}=\int_{0}^{\infty} Q_{M / 2}\left(\sqrt{h^{2} \eta_{E} \gamma / \sigma^{2}}, \sqrt{\lambda / \sigma^{2}}\right) f_{\gamma}(\lambda) \mathrm{d} \gamma
$$

and by integrating (26), an enhanced closed-form expression of detection probability using compressed measurements over Rayleigh fading channel, $\bar{P}_{D B C M}$, using Eq B.53 in [36] could be expressed as:

$$
\bar{P}_{D B C M}=\mathrm{e}^{\left(-\frac{\lambda}{2 \sigma^{2}}\right)} \sum_{k=0}^{l} \frac{1}{k !}\left(\frac{\lambda}{2 \sigma^{2}}\right)^{k}+\left(\frac{1+u}{u}\right)^{l}\left(\mathrm{e}^{\left(-\frac{\lambda}{2(1+u) \sigma^{2}}\right)}-\mathrm{e}^{\left(-\frac{\lambda}{2 \sigma^{2}}\right)} \sum_{k=0}^{l} \frac{1}{k !}\left(\frac{\lambda u}{2(1+u) \sigma^{2}}\right)^{k}\right)
$$

where $l=M / 2-1$ and $\mu=h^{2} \eta_{E} \gamma$.

\section{Simulation Results and Discussions}

In this section, we illustrate the simulation results of the performance of Enhanced Compressed Measurement Based Energy Detection (ECMBED). The illustration could be categorized into two parts: a) the simulation results of testing the performance of ECMBED in comparison with the closed form of (27) over Rayleigh fading channel and to the achievement results in [31], and Compressed detector, as well. b) ECMBED performance over Rayleigh fading channel is compared to the result in [17], (see Fig. 1 within the ref. [17]).

In both cases, the PU transmitted signal follows the IEEE802.22 WRAN standard. It is an OFDM signal with $2048 \mathrm{FFT}, 4.45 \mathrm{Mbps}$ data rate, $f_{c}$ is $54 \mathrm{MHz}$, and 16QAM payload modulation. The receiver operating characteristic (ROC) curves analysis has commonly been employed in the signal detection theory. It is a method to measure the tradeoff between the detection probability or the miss-detection probability with respect to the false alarm probability.

In Fig 2, the performance of ECMBED versus SNR at different probabilities of false alarm values with $M / N=0.2$ is depicted. In other words, 20 percentage of the collected number of samples $N$ is used only to detect PU transmitted signal on the observed frequency band. The result revealed that the performance of detection probability improves as the probability of false alarm increases to the limit assigned by IEEE802.22 WRAN. For example, 
almost $90 \%$ of detection could be achieved using $-10 \mathrm{~dB}$ signal-to-noise ratio with $P_{F}=0.1$ while $P_{D}=0.62$ is achieved with $P_{F}=0.01$. Likewise, $P_{D}=0.9$ could also be achieved at $P_{F}=0.01$ with only $3 \mathrm{~dB}$ marginal signal-tonoise ratio. Furthermore, at low value of probability of false alarm e.g., $P_{F}=0.01$, the marginal SNR required to shift the probability of detection $P_{D}$ from $50 \%$ to $90 \%$ has been quite small.

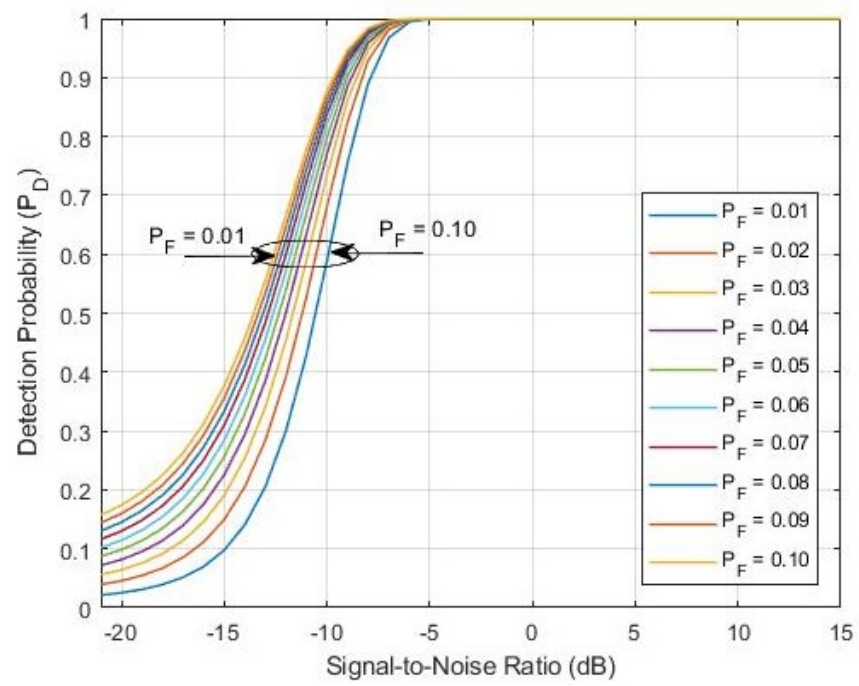

Figure 2 Performance of ECMBED at different $P_{F}$, with $M / N=20 \%$

\subsection{ECMBED Performance in Comparison with Closed Form}

In this subsection, we studied the performance trade-off between $P_{M}$ and $P_{F}$ over Rayleigh fading channel and depicted the results using complementary ROC curves as in Fig. 3. As shown that the performance of the proposed analytical expression of $P_{D, R B C M}$ produced sustainable result when it is compared to simulated ECMBED algorithm. The difference is barely noticed and the curves of $P_{D, R B C M}$ showed closeness in results.

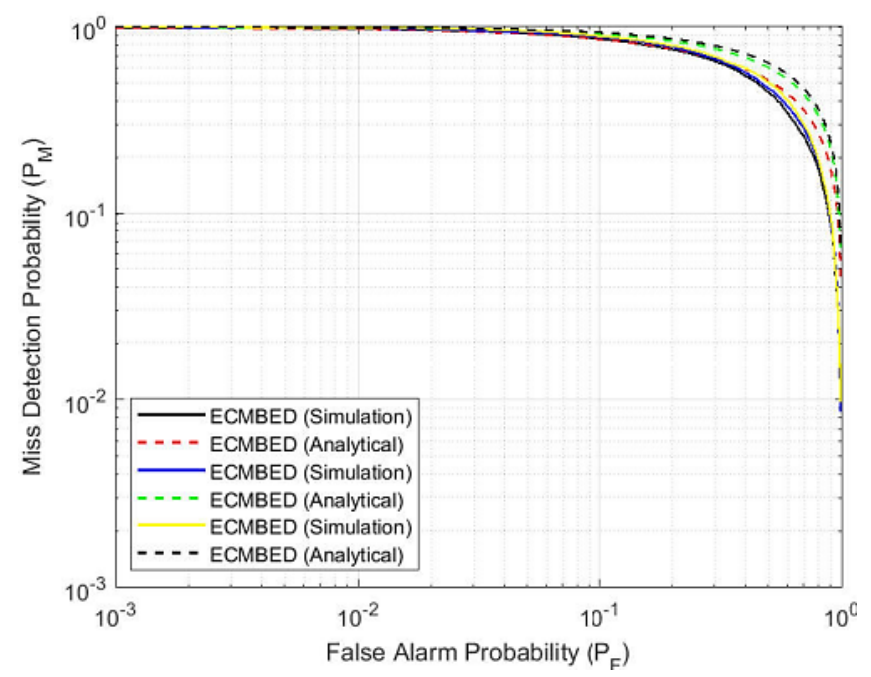

Figure 3 Complementary ROC curves of $P_{D, R B C M}$ comparing to simulated ECMBED results over Rayleigh fading channel $(\gamma=-10,-15$ and $-20 \mathrm{~dB})$

\subsection{ECMBED Performance in Comparison with Compressed Detector}

In this subsection, we tested the detection performance of ECMBED using different values of SNR. The obtained results have been compared to the results achieved by the compressed detection as shown in Fig. 4. The simulated results showed that ECMBED produced high detection performance in comparison with compressed detector using only 20 percentage of compression ratio.

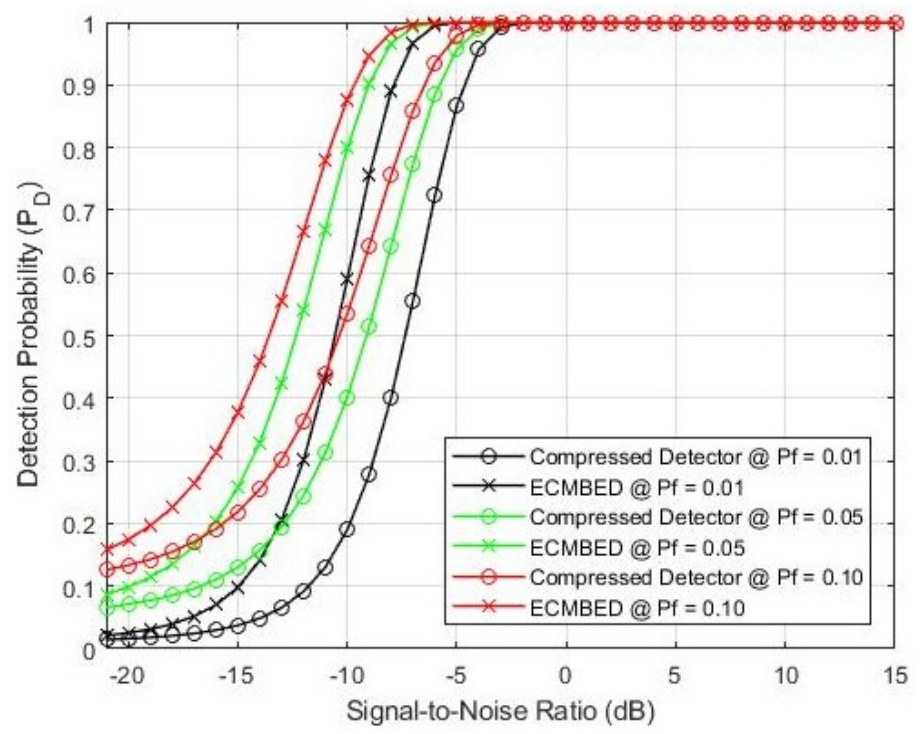

Figure 4 Performances comparison between ECMBED and compressed detector at different $P_{F}, M / N=20 \%$ and $N=100$.

Also we have reduced the compression ratio to be in magnitude of 10 percentage and observed the detection performance using different amount of probability of false alarm. Even if the compression ratio reduction is $10 \%$, ECMBED showed better performance and the results illustrated in Fig. 5.

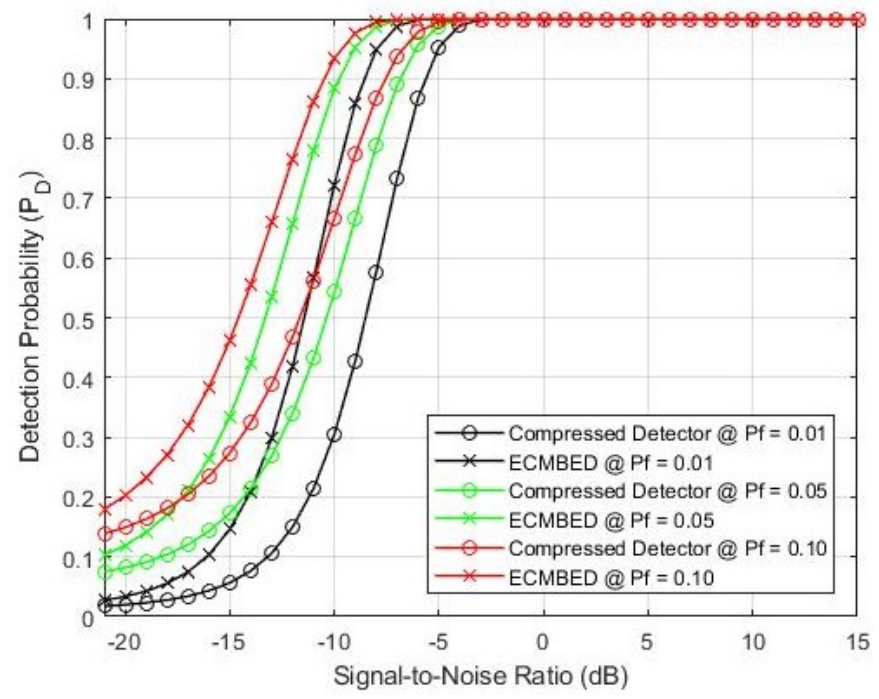

Figure 4 Performances comparison between ECMBED and compressed detector at different $P_{F}, M / N=10 \%$ and $N=100$. 
Fig. 4 and 5 illustrated the impact of using compressed measurements. As it has been realized that compressed measurements has permitted the SU to sense the PU signal even if we used 10 percentage of the ratio of compression. In other words, ECMBED used small amount of samples to decide the status of the observed frequency band, occupied or not which considered far less than that needed when Nyquist sampling rate has been applied. Finally, we also tested that performance of ECMBED using complementary ROC curves and compared the obtained results to the results obtained by ordinary energy detection (OED), CMBED and the compressed detector. The comparison parameters were $M / N=20 \%, N=100$ and $S N R=-15 \mathrm{~dB}$. The simulation results of ECMBED performance were high as depicted in Fig. 6.

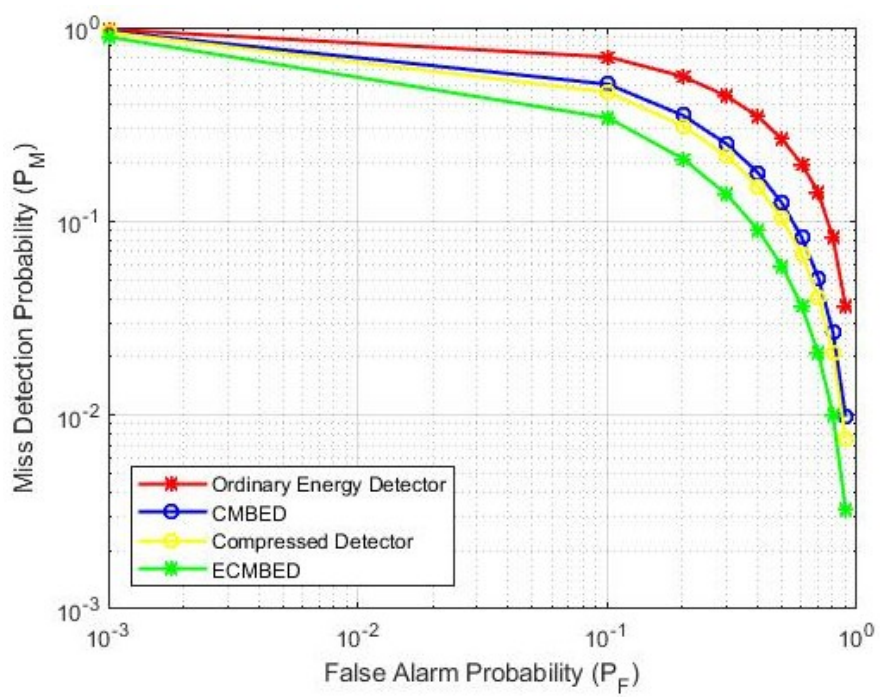

Figure 6 Complementary ROC curves of ECMBED, $\mathrm{OED}, \mathrm{CMBED}$ and the compressed detector at $\mathrm{SNR}=-15$ $\mathrm{dB}$ and $N=100$.

We also studied the effect of $\eta_{E}$ on the mis-detection probability versus threshold. and it has been realized that $\eta_{E}$ affected the choice of detection threshold as depicted in Fig. 7. However the improving introduced by $\eta_{E}$ would be at the expense of scarifying the reduction in compression ratio. Therefore, the increase in the magnitude of $\eta_{E}$ should be bounded to the point that compressed measurements still valid.

For more clarity to Fig. 7, the trade-off between $\eta_{E}$ and $M /$ Nhas been depicted in Fig. 8. It has been realized that as $M / N$ increased the value of $\eta_{E}$ increased too, however in the same figure the detection performance after certain value of $M / N$, the performance of the probability of detection degraded.

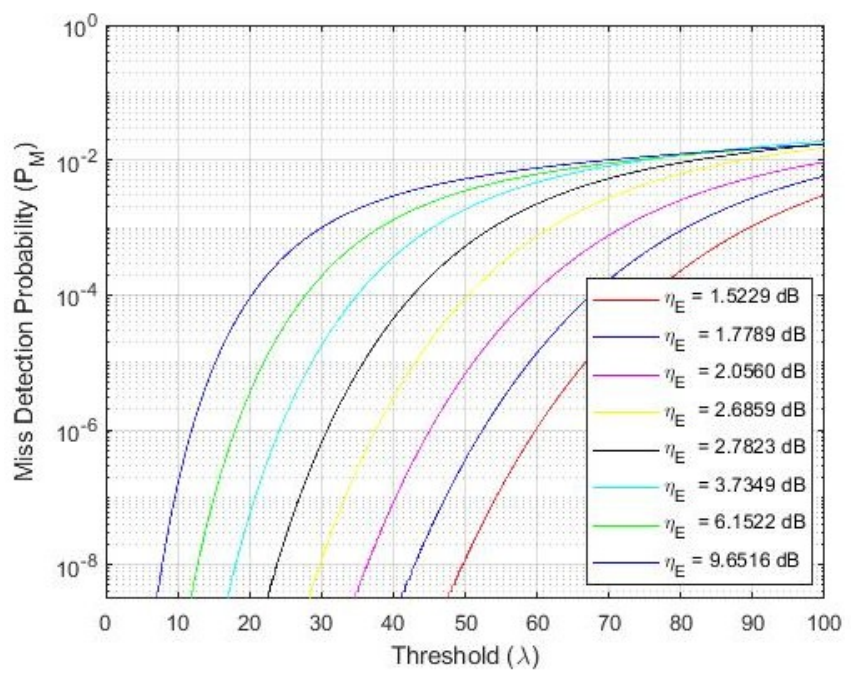

Figure 7 The effect of $\eta_{E}$ on the detection threshold

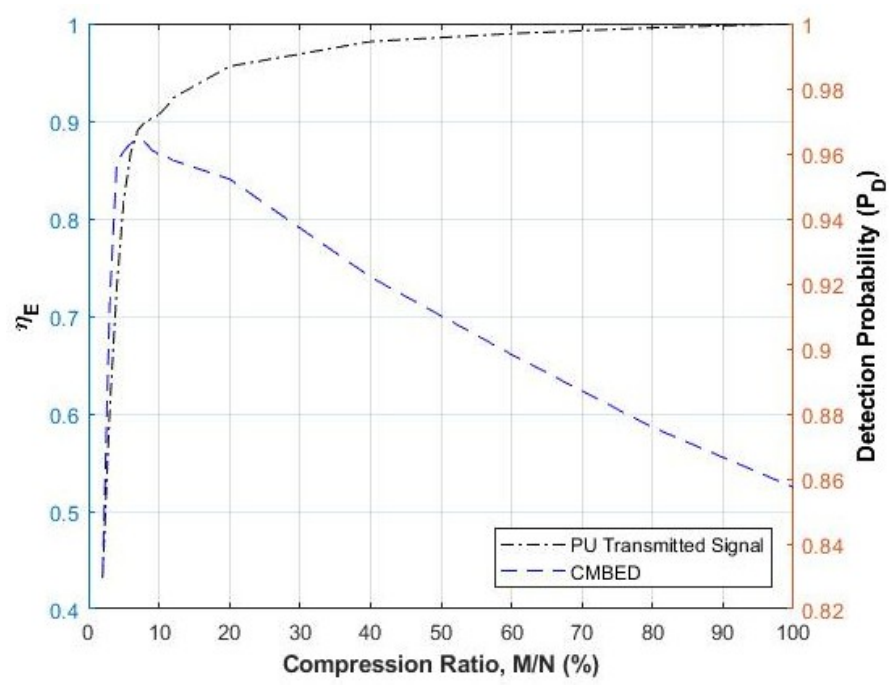

Figure8the effect of compression ratios on both detection performance of ECMBED and the fraction of PU transmitted signal.

\subsection{ECMBED Performance over Rayleigh Fading Channel}

ECMBED complementary ROC curves over Rayleigh channel when $M=4$ and $M=10$ is illustrated in Fig. 9, in both cases, ECMBED shows better performance than the OED over fading channel as in [17]. 

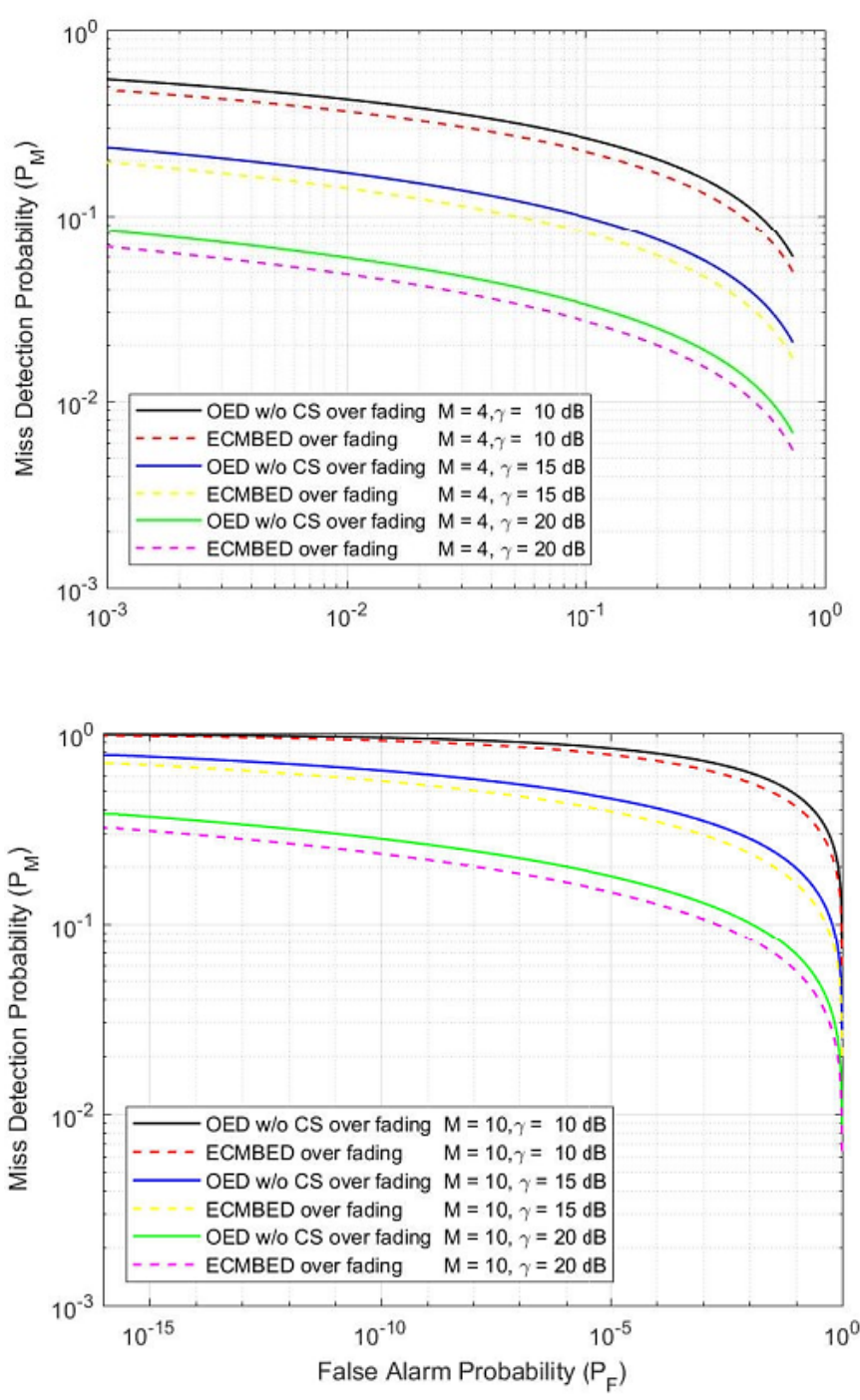

Figure 9 Comparing ECMBED performance results to the result obtained in [17] over Rayleigh fading channel using complementary ROC curve, (Top figure, $\mathrm{M}=4$, bottom figure $\mathrm{M}=10$ ).

Finally, we investigated the effect of SNR on the performance of analytical expression of (27) as in Fig. 10.

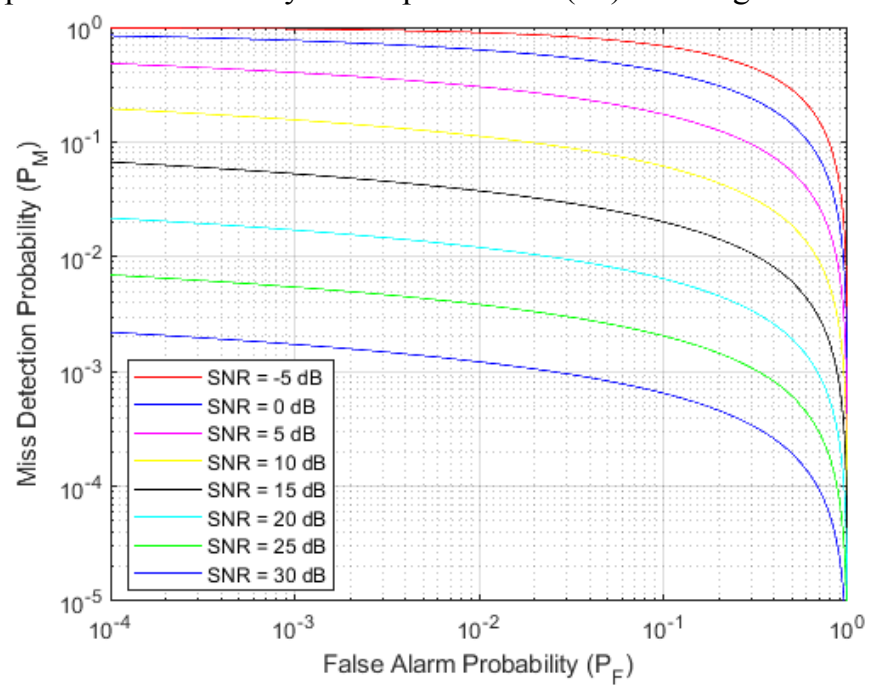

Figure 10 the effect of SNR on the performance of the analytical expression of (27).

\section{Conclusion}

In this paper, we used compressed sensing to emphasize that despite the reduction of using a small number of measurements $M(M<<N)$, the energy detector using compressed measurements can obviously able to detect the presence of PU transmitted signal on the frequency band of interest. Comparing to the traditional case without the use of compressed measurements, the energy detector requires large number of measurements $M(M \rightarrow N)$ to detect the existing of PU signal on that channel under observation. By result, using less measurements $M$ for detection is much better than using large $N$ in same case in which this reduction contribute implicitly faster sensing with less time. In addition to that using less measurements in traditional energy detector may cause falsely declaring the observed channel unoccupied while it is not and yields harmful interference to the network. In this paper, the performance of compressed energy detection based compressive spectrum sensing over Rayleigh fading channel has been investigated. It has been observed that even though the collected number of samples for detection is less than the number required by Nyquist criterion, the proposed method could detect the PU transmitted signal at a high compression ratio $M / N=10 \%$. The impact of using fraction of transmitted signal and the compression ratios on the performance of detection and threshold has also been investigated. When we compared the performance of ECMBED closed form to that realized by simulation, the results were close in both cases, and outperforms the results obtained by ordinary energy detection and compressed detection. The overall detector performance has been affected by the SNR values which is attributed to intrinsic variations of the corresponding detection probability.

\section{References}

[1] T. Yucek and H. Arslan, "A survey of spectrum sensing algorithms for cognitive radio applications," IEEE communications surveys \& tutorials, vol. 11, no. 1, pp. 116-130, 2009.

[2] M. Abo-Zahhad, S. M. Ahmed, M. Farrag, and K. A. BaAli, "Wideband Cognitive Radio Networks Based Compressed Spectrum Sensing: A Survey," Journal of Signal and Information Processing, vol. 9, p. 122, 2018.

[3] Q. Zhao and B. M. Sadler, "A survey of dynamic spectrum access," Signal Processing Magazine, IEEE, vol. 24, no. 3, pp. 79-89, 2007.

[4] F. S. P. T. Force, "Report of the spectrum efficiency working group," ed: Nov, 2002.

[5] Z. Lei and S. J. Shellhammer, "IEEE 802.22: The first cognitive radio wireless regional area network standard," IEEE communications magazine, vol. 47, no. 1, pp. 130-138, 2009.

[6] S. Foucart and H. Rauhut, A mathematical introduction to compressive sensing (no. 3). Birkhäuser Basel, 2013.

[7] E. J. Candè and M. B. Wakin, "An introduction to compressive sampling," Signal Processing Magazine, IEEE, vol. 25, no. 2, pp. 21-30, 2008

[8] H. Urkowitz, "Energy detection of unknown deterministic signals," Proceedings of the IEEE, vol. 55, no. 4, pp. 523-531, 1967.

[9] M. López-Benítez and F. Casadevall, "Improved energy detection spectrum sensing for cognitive radio," IET communications, vol. 6, no. 8, pp. 785-796, 2012.

[10] X. Xie and X. Hu, "Improved energy detector with weights for primary user status changes in cognitive radios networks," in Consumer Communications and Networking Conference (CCNC), 2014 IEEE 11th, 2014, pp. 53-58: IEEE. 
[11] J. Shen, S. Liu, Y. Wang, G. Xie, H. F. Rashvand, and Y. Liu, "Robust energy detection in cognitive radio," IET communications, vol. 3, no. 6, pp. 1016-1023, 2009.

[12] K. Arshad and K. Moessner, "Robust spectrum sensing based on statistical tests," Iet Communications, vol. 7, no. 9, pp. 808-817, 2013.

[13] J. Song, Z. Feng, P. Zhang, and Z. Liu, "Spectrum sensing in cognitive radios based on enhanced energy detector," IET communications, vol. 6, no. 8, pp. 805-809, 2012.

[14] E. Abdessamad, R. Saadane, M. El Aroussi, M. Wahbi, and A Hamdoun, "Spectrum sensing with an improved Energy detection," in Multimedia Computing and Systems (ICMCS), 2014 International Conference on, 2014, pp. 895-900: IEEE.

[15] A. Taherpour, S. Gazor, and M. Nasiri-Kenari, "Wideband spectrum sensing in unknown white Gaussian noise," IET communications, vol. 2 , no. 6, pp. 763-771, 2008

[16] O. Altrad and S. Muhaidat, "A new mathematical analysis of the probability of detection in cognitive radio over fading channels," EURASIP Journal on Wireless Communications and Networking, vol. 2013, no. 1, p. 159, 2013.

[17] F. F. Digham, M.-S. Alouini, and M. K. Simon, "On the energy detection of unknown signals over fading channels," IEEE transactions on communications, vol. 55, no. 1, pp. 21-24, 2007.

[18] V. I. Kostylev, "Energy detection of a signal with random amplitude," in Communications, 2002. ICC 2002. IEEE International Conference on, 2002, vol. 3, pp. 1606-1610: IEEE.

[19] F. B. de Carvalho, W. T. Lopes, and M. S. Alencar, "Performance of Cognitive Spectrum Sensing Based on Energy Detector in Fading Channels," Procedia Computer Science, vol. 65, pp. 140-147, 2015.

[20] I. E. Atawi, O. S. Badarneh, M. S. Aloqlah, and R. Mesleh, "Spectrum-sensing in cognitive radio networks over composite multipath/shadowed fading channels," Computers \& Electrical Engineering, vol. 52, pp. 337-348, 2016.

[21] A.-A. A. Boulogeorgos, N. D. Chatzidiamantis, and G. K. Karagiannidis, "Spectrum sensing with multiple primary users over fading channels," IEEE Communications Letters, vol. 20, no. 7, pp. 1457-1460, 2016.

[22] J. Li, B. Li, and M. Liu, "Performance analysis of cooperative spectrum sensing over large and small scale fading channels," AEU-International Journal of Electronics and Communications, vol. 78, pp. 90-97, 2017.

[23] V. M. Patil, R. Ujjinimatad, and S. R. Patil, "Signal Detection in Cognitive Radio Networks over AWGN and Fading Channels," International Journal of Wireless Information Networks, vol. 25, no. 1 , pp. 79-86, 2018

[24] H. Sun, D. I. Laurenson, and C.-X. Wang, "Computationally tractable model of energy detection performance over slow fading channels," IEEE Communications Letters, vol. 14, no. 10, pp. 924-926, 2010

[25] A. Rao and M.-S. Alouini, "Performance of cooperative spectrum sensing over non-identical fading environments," IEEE Transactions on Communications, vol. 59, no. 12, pp. 3249-3253, 2011.

[26] K. Ruttik, K. Koufos, and R. J'antti, "Detection of unknown signals in a fading environment," IEEE communications letters, vol. 13 , no. $7,2009$.

[27] P. C. Sofotasios, E. Rebeiz, L. Zhang, T. Tsiftsis, D. Cabric, and S. Freear, "Energy detection based spectrum sensing over and extreme fading channels," Vehicular Technology, IEEE Transactions on, vol. 62, no. 3, pp. 1031-1040, 2013.

[28] V. R. S. Banjade, N. Rajatheva, and C. Tellambura, "Performance analysis of energy detection with multiple correlated antenna cognitive radio in Nakagami-m fading," IEEE Communications Letters, vol. 16, no. 4, pp. 502-505, 2012.

[29] D. A. Shnidman, "The calculation of the probability of detection and the generalized Marcum Q-function," IEEE Transactions on Information Theory, vol. 35, no. 2, pp. 389-400, 1989.

[30] M. A. Davenport, M. B. Wakin, and R. G. Baraniuk, "Detection and estimation with compressive measurements," Dept. of ECE, Rice University, Tech. Rep, 2006.

[31] T. A. Khalaf, M. Y. Abdelsadek, and M. Farrag, "Compressed measurements based spectrum sensing for wideband cognitive radio systems," International Journal of Antennas and Propagation, vol. 2015, 2015.
[32] Abo-Zahhad, M., Sabah M. Ahmed, Mohammed Farrag, and Khaled Ali BaAli. "Detection of primary user signal in wideband cognitive radio networks exploiting DCT as sensing matrix." In Radio Science Conference (NRSC), 2017 34th National, pp. 152-159. IEEE, 2017.

[33] A. V. Oppenheim, Discrete-time signal processing. Pearson Education India, 1999.

[34] R. Ujjinimatad and S. R. Patil, "Mathematical analysis for detection probability in cognitive radio networks over wireless communication channels," The Journal of Engineering, vol. 1, no. 1,2014

[35] M. K. Simon and M.-S. Alouini, Digital communication over fading channels. John Wiley \& Sons, 2005.

[36] M. K. Simon, Probability distributions involving Gaussian random variables: A handbook for engineers and scientists. Springer Science \& Business Media, 2007. 